\title{
Fractal Analysis of Discharge Current Fluctuations
}

\author{
S. Kimiagar ${ }^{1}$, M. Sadegh Movahed $^{2}$, S. Khorram ${ }^{3}$, S. Sobhanian ${ }^{4,5}$, and M. Reza Rahimi Tabar ${ }^{6,7,8}$ \\ ${ }^{1}$ Department of Physics,science faculty, Tehran central branch, Islamic Azad University, Tehran, Iran \\ ${ }^{2}$ Department of Physics, Shahid Beheshti university, G.C., Evin, Tehran 19839, Iran \\ 3 Research Institute for Applied Physics and Astronomi, University of Tabriz, Tabriz 51664, Iran \\ ${ }^{4}$ Department of Theoretical Physics and Astrophysics, Tabriz University, Tabriz 51664, Iran \\ ${ }^{5}$ Research Institute for Fundamental Sciences, Tabriz 51664, Iran \\ ${ }^{6}$ Department of Physics, Sharif University of Technology, P.O.Box 11365-9161, Tehran, Iran \\ ${ }^{7}$ CNRS UMR 6529, Observatoire de la Côte d'Azur, BP 4229, 06304 Nice Cedex 4, France \\ ${ }^{8}$ Carl von Ossietzky University, Institute of Physics, D-26111 Oldenburg, Germany
}

\begin{abstract}
We use the multifractal detrended fluctuation analysis (MF-DFA) to study the electrical discharge current fluctuations in plasma and show that it has multifractal properties and behaves as a weak anti-correlated process. Comparison of the MF-DFA results for the original series with those for the shuffled and surrogate series shows that correlation of the fluctuations is responsible for multifractal nature of the electrical discharge current.
\end{abstract}

\section{INTRODUCTION}

Plasma physics is concerned with the complex interaction of many charged particles with external or selfgenerated electromagnetic fields. It plays an essential role in many applications, ranging from advanced lighting devices to surface treatments for semiconductor applications or surface layers. At the same time, the interpretation and estimation of physical and chemical properties of a plasma fluid have been one of the main research areas in the science of magnetohydrodynamics and transport theory [1-15]. As in many other fields in physics, the complex physics requires advanced numerical tools to be developed and used.

It is well-known that the discharge current fluctuations in the plasma often exhibit irregular and complex behavior. Indeed, the current fluctuations represent a dynamical system influenced by many factors, such as the pressure, the electrical potential between cathode and anode, the electrical properties of the gas, noises, and trends, due to the experimental setup, etc. Factors that influence the trajectory of discharge current fluctuations have enormously large phase space. Thus, the use of stochastic tools for investigating their statistical properties is natural. Because of the complexity and stochasticity of the discharge fluctuations in the plasma fluid, it is generally difficult to have access to detailed dynamics of the plasma ions, without paying attention to the statistical aspect of plasma. Therefore, there may be no remedy, except using stochastic analysis to investigate the evolution and physical properties of the discharge current produced by such fluctuations. Also due to the limitations in the experimental setup for measuring the fluctuations, as well as the finiteness of the available data in some cases, the original fluctuations may be affected by some trends and non-stationarities. Therefore, in order to infer valuable statistical properties of the original fluctuations and avoid spurious detection of correlations, one must use a robust method which should be insensitive to any trends.

Fluctuation of the electric and magnetic fields of plasma, spectral density, logistic mapping and nonlinearity of ionization wave have been investigated in [1-14]. Recently, Carreras et. al., have shown that the plasma has a multifractal nature with intermittency levels comparable to the levels measured in neutral fluid turbulence [14]. Also, Budaev et. al., used the scaling behavior of structure functions and wavelet transform modulus maxima (WTMM). They showed the anomalous transport of particles in the plasma phase attached to the turbulent property has multifractal nature [8,11-13].

Although the analysis of discharge current in the plasma has a long history [16-18], nevertheless, some important issues, such as ions and electrons acceleration mechanism, interaction between laser and plasma, especially from the statistical properties point of view, fractal features, effects of trends in small and large scales and the kind of correlations have remained unexplained [1-17].

Generally, correlated and uncorrelated time series could have same probability distribution function. Also they may have mono-fractal or multi-fractal nature. The mono-fractal signals can be describe by one scaling exponent. However many time series do not exhibit a simple monofractal scaling behavior. In some cases, there exist crossover (time-) scales separating regimes with different scaling exponents. In other cases, the scaling behavior is more complicated, and different scaling exponents are required for different parts of the series. This occurs, e. g., when the scaling behavior in the first half of the series differs from the scaling behavior in the second half. In even more complicated cases, such different scaling 
behavior can be observed for many interwoven fractal subsets of the time series. In this case a multitude of scaling exponents is required for a full description of the scaling behavior, and a multifractal analysis must be applied. In nature, two different types of multifractality in time series can be distinguished: (i) Multifractality due to a broad probability density function for fluctuations. In this case the multifractality cannot be removed by shuffling the series. (ii) Multifractality due to different (time-) correlations for small and large fluctuations. If both kinds of multifractality are present, the shuffled series will show weaker multifractality than the original series.

Here we rely on the state-of-the-art of computational methods in statistical physics to characterize the complex behavior of electrical discharge time series. We study the discharge current fluctuations (see the upper panel of Figure 1) by the multifractal detrended fluctuation analysis (MF-DFA) and Fourier-detrended fluctuation analysis (F-DFA) methods. Using the method proposed in $[19,20]$, we investigate the relation between the amplitude and the period of the trend and crossover in discharge current fluctuations in the plasma.

The rest of this paper is organized as follows: In Section II we describe some important steps of fractal analysis to explore the stochastic time series in the presence of sinusoidal trends. The Hurst exponent and its relation to the classical multifractal, the generalized multifractal dimension and the Hölder exponents are described in section II. Data preparation, experimental setup, surrogate and shuffled time series are also given in section II. We then eliminate the sinusoidal trends via the F-DFA technique in section III, and investigate the multifractal properties of the remaining fluctuations. In section IV we deal with the source of multifractality in data. Section $\mathrm{V}$ is devoted to a summary of the results.

\section{ANALYSIS TECHNIQUES AND EXPERIMENTAL SETUP}

The simplest type of multifractal analysis is based upon the standard partition function, which has been developed for multifractal characterization of normalized, stationary measurements [21-24]. The standard formalism does not yield the correct results for nonstationary time series that are affected by trends. The improved multifractal formalism has been developed by Muzy et al. [25], which is known as the wavelet-transform modulus maxima (WTMM) method $[25,26]$. It is based on the wavelet analysis and involves tracing the maxima lines in the continuous wavelet transform over all the scales. The second method which is known as MF-DFA, is based on the identification of the scaling behavior of the $q$ th moments and is the generalization of the standard DFA which uses only the second moment, $q=2[19,20,27-32]$.

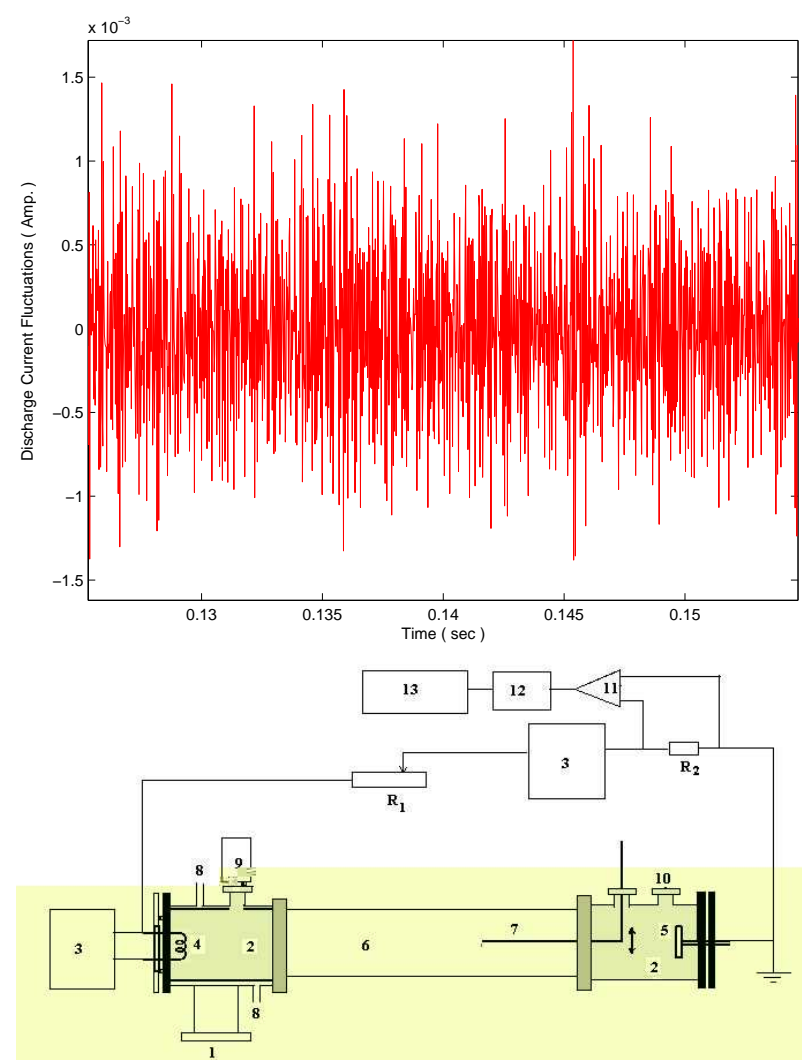

FIG. 1. Upper panel: typical discharge current fluctuations as a function of time in our experimental setup. Lower panel: the sketch of the experimental setup to record the discharge current fluctuations in the tube, filled by helium, with (1) vacuum pumps; (2) copper cylinder; (3) power supply; (4) hot cathode; (5) anode plate; (6) glass tube; (7) single Langmuir probe; (8) water cooling; (9) Pirani pressure gage; (10) gas inlet; (11) OP-Amp; (12) low pass filter, and (13) A/D card and $\mathrm{PC}$.

It has successfully been applied to diverse problems, such as heart rate dynamics [33-35], economical time series [36-41], river flow [42] and sunspot fluctuations [43], cosmic microwave background radiations [44], and music [45-47].

In general, experimental data are often affected by non-stationarities, such as trends which must be welldistinguished from the intrinsic fluctuations of the series, in order to determine their correct scaling behavior. In addition, very often we do not know the reasons for the underlying trends in the collected data and, even worse, we do not know the scales of the underlying trends. For reliable detection of the correlations, it is essential to distinguish trends from the intrinsic fluctuations in data. Hurst rescaled-range analysis [48] and other non-detrending methods work well if the records are long and do not involve trends. But, if trends are present in the data, they might yield inaccurate results. In general, the MF-DFA is a well-established method for determining the scaling behavior of noisy data in 
the presence of trends without knowing their origin and shape $[27,28,33,49,50]$. Here in order to eliminate the effect of sinusoidal trend, we apply the Fourier DFA (FDFA) [51,52]. After elimination of the trend we use the MF-DFA to analyze the data set.

\section{A. MF-DFA Method}

The MF-DFA consists of the following four steps (see [19,20,27,29-32,53] for more details):

(i): Computing the profile of underlying data series, $x_{k}$, as

$$
Y(i) \equiv \sum_{k=1}^{i}\left[x_{k}-\langle x\rangle\right] \quad i=1, \ldots, N
$$

(ii): Dividing the profile into $N_{s} \equiv \operatorname{int}(N / s)$ nonoverlapping segments of equal lengths $s$, and then computing the fluctuation function for each segment

$$
F^{2}(s, m) \equiv \frac{1}{s} \sum_{i=1}^{s}\left\{Y[(m-1) s+i]-y_{m}(i)\right\}^{2}
$$

where $y_{m}(i)$ is a fitting polynomial in segment $m$ th. Usually, a linear function is selected for fitting the function. If there do not exist any trends in the data, a zerothorder fitting function might be enough [27,28,35].

(iii): Averaging the local fluctuation function over all the part, given by

$$
F_{q}(s) \equiv\left\{\frac{1}{N_{s}} \sum_{m=1}^{N_{s}}\left[F^{2}(s, m)\right]^{q / 2}\right\}^{1 / q}
$$

Generally, $q$ can take any real value, except zero. For $q=2$, the standard DFA procedure is retrieved.

(iv): The final step is determining the slope of the log$\log$ plot of $F_{q}(s)$ versus $s$ directly determines the so-called generalized Hurst exponent $h(q)$, as

$$
F_{q}(s) \sim s^{h(q)}
$$

For stationary time series, such as the fractional Gaussian noise (fGn), $Y(i)$ in Eq. (1) will be a fractional Brownian motion (fBm), and so, $0<h(q=2)<1.0$. The exponent $h(2)$ is identical with the well-known Hurst exponent $H$ [21,27-29]. Moreover, for a nonstationary series, such as the $\mathrm{fBm}, Y(i)$ in Eq. (1) will be a sum of the $\mathrm{fBm}$ series and, thus, the corresponding scaling exponent of $F_{q}(s)$ is identified by $h(q=2)>1.0[27,28,54]$ (see the appendix of $[42,43]$ for more details). In this case, the relation between the exponent $h(2)$ and $H$ is $H=h(q=2)-1$. The auto-correlation function is characterized by a power law, $C(s) \equiv\left\langle n_{k} n_{k+s}\right\rangle \sim s^{-\gamma}$, with $\gamma=2-2 H$. Its power spectrum is given by, $S(\nu) \sim \nu^{-\beta}$, with frequency $\nu$ and $\beta=2 H-1$. In the nonstationary case, the correlation function is

$$
C(i, j)=\left\langle x_{i} x_{j}\right\rangle \sim i^{2 H}+j^{2 H}-|i-j|^{2 H}
$$

where $i, j \geq 1$ and the power-spectrum scaling exponent is, $\beta=2 H+1[27,28,42,43,54]$.

For monofractal time series, $h(q)$ is independent of $q$, since the scaling behavior of the variances $F^{2}(s, m)$ is identical for all the segments $m$, and the averaging procedure in Eq. (3) will just yield identical scaling behavior for all values of $q$. If we consider positive values of $q$, the segments $m$ with large variance $F^{2}(s, m)$ (i.e., large deviations from the corresponding fit) will dominate the average $F_{q}(s)$. Thus, for positive values of $q, h(q)$ describes the scaling behavior of the segments with large fluctuations. For negative values of $q$, on the other hand, the segments $m$ with small variance $F^{2}(s, m)$ will dominate in the average $F_{q}(s)$, and $h(q)$ describes the scaling behavior of the segments with small fluctuations.

The classical multifractal scaling exponents $\tau(q)$, defined by the standard partition function-based formalism, discussed in literature [21-24,30-32,43], is related to the generalized hurst exponent via the MF-DFA as

$$
\tau(q)=q h(q)-1
$$

Moreover, the generalized multifractal dimensions $D(q)$ read as

$$
D(q) \equiv \frac{\tau(q)}{q-1}=\frac{q h(q)-1}{q-1}
$$

Another way of characterizing a multifractal series is through its singularity spectrum $f(\alpha)$, which is related to $\tau(q)$ via a Legendre transform $[21,23]$. Here, $\alpha$ is the singularity strength or the Hölder exponent. Using Eq. (6), we can directly relate $\alpha$ and $f(\alpha)$ to $h(q)$

$$
\alpha=h(q)+q h^{\prime}(q) \quad \text { and } \quad f(\alpha)=q[\alpha-h(q)]+1
$$

A single Hölder exponent denotes monofractality, while in the multifractal case, different parts of the structure are characterized by different values of $\alpha$, leading to the existence of the spectrum $f(\alpha)$.

In some cases, there exist one or more crossover (time) scales, $s_{\times}$, segregating regimes with different scaling exponents, e.g., the correlation exponent for $s \ll s_{\times}$and another type of the correlation or uncorrelated behavior for $s \gg s_{\times}[19,20,30-32]$. In the presence of different behavior of the various moments in the MF-DFA method, distinct scaling exponents are required for different parts of the series $[19,20]$. Therefore, one needs a multitude of scaling exponents (multifractality) for a full description of the scaling behavior. A crossover usually can arise from a change in the correlation properties of the signal at different time or space scales, or can often arise from trends in the data $[19,20]$. However it is well-known 
WTMM method can remove this crossover but, in many case, the presence of crossover as well as their values have physical importance.

Let us mention two advantages of the MF-DFA method in order to compare with the applicability of WTMM method. The first advantage corresponds to the effort of programming and second one is related to the performance and reliability of given results. The MF-DFA does not require the modulus maxima procedure while WTMM need to do this task. The wavelet coefficients can become arbitrary small in WTMM method. Subsequently MF-DFA does not involve more effort in programming as well as more time consuming in contrast to WTMM, specially for long length time series such as our underlying data set [53]. The second advantage is related to the fact that the MF-DFA gives more reliable results than WTMM specially, for negative moments. It has been reported that WTMM gives an overestimated multifractal exponent and in some cases WTMM can also give different results if one applies different wavelets [55]. However, the most disadvantage and limitation of MFDFA method will appear when it is applied to investigate data set with small size. In this case MF-DFA gives rich singularity spectrum corresponds to more multifractality property than what should be existed. Fortunately this circumstance does not occur in our situation, because the typical size of our plasma data is about $10^{6}$.

In order to remove the trends correspond to the low frequency periodic behavior, we transform the recorded data to the Fourier space using the method proposed in [56] (see also [19,20,30-32]). Using this method we can track the influence of sinusoidal trends on the results and determine the value of so-called crossover in the fluctuation function, in terms of the scale in DFA method. We determine over which scale noises or trends have dominant contribution $[51,52,57,58]$. After removing the dominant periodic functions, such as sinusoidal trends, we obtain the fluctuation exponent by direct application of the MF-DFA. If truncation of the number of the modes be sufficient, the crossover due to a sinusoidal trend in the log-log plot of $F_{q}(s)$ versus $s$ disappear.

\section{B. Data description and experimental setup}

To investigate the stochastic nature of the discharge current fluctuations in a typical plasma, we constructed an experimental setup shown in the lower panel of Figure 1. The discharge glass tube has two copper ends, $80 \mathrm{~mm}$ in diameter and $110 \mathrm{~cm}$ in length. One end is the anode electrode (a flat copper plate as a positive pole), while the other end represents the cathode (tungsten filament as a negative pole and electron propagator). The discharge tube is evacuated to a base pressure of 0.1 up to 0.8 torr under a voltage of $400-900 \mathrm{~V}$ and filled with Helium as the working gas. The pressure, voltage and current should be optimal for ensuring the stability of the plasma. The discharge current fluctuations were monitored using a resistor which was connected to an operational amplifier impedance converter. We fixed the pressure and investigated how the statistical properties of plasma changing under variation of the current. The fluctuations of the discharge current were digitized and cleaned with a filter that omitted direct current. Thereafter, the fluctuation of the discharge were recorded at a rate of 44100 sample/sec, with a resolution of 12 bits, using a analogdigital card for several values of the electrical discharge current intensity, namely, 50,60,100,120,140,180, and $210 \mathrm{~mA}$. The typical size of the recorded data for every current intensity is about $10^{6}$.

\section{Surrogate and shuffled data}

The phase-randomized surrogate consists of three steps [59-62]:

(i) Computing the discrete fourier transform (DFT) coefficients of the series

$\mathcal{F}^{2}\{x(t)\} \equiv|X(\nu)|^{2}=|X(k)|^{2}=\left|\frac{1}{\sqrt{N}} \sum_{n=0}^{N-1} x\left(t_{n}\right) e^{i 2 \pi n k / N}\right|^{2}$

where $\nu=k / N \Delta t$ and $\Delta t$ is the step of digitalization in the experimental setup.

(ii) Multiplying the DFT coefficients of the series by a set of pseudo-independent, uniformly distributed $\phi(\nu)$ quantities in the range $[0,2 \pi)$

$$
\tilde{X}(\nu)=X(\nu) e^{i \phi(\nu)}
$$

(iii) The surrogate data set is given by the inverse DFT as

$$
\mathcal{F}^{-1}\{\tilde{X}(\nu)\} \equiv \tilde{x}\left(t_{n}\right)=\frac{1}{\sqrt{N}} \sum_{k=0}^{N-1}\left|X_{k}\right| e^{i \phi(k)} e^{-i 2 \pi n k / N}
$$

The power spectrum of the surrogate data set is the same as one for the original data. According to the Wiener-Khintchine theorem, surrogate data has the same autocorrelation as the initial series $[59,60]$. The amplitude of the surrogate data will be preserved as in the original data. However the probability density function of the data will change to the Gaussian distribution. We note that applying the DFT needs the data to be periodic [63]. In addition, this procedure eliminates nonlinearities, preserving only the linear properties of the underlying original data set [60].

To produce a shuffled data set, one should clean the imposed memory in series. To this end, we should randomize the order of data in underlying series while their values remain unchanged. 


\section{FRACTAL ANALYSIS OF ELECTRICAL DISCHARGE FLUCTUATIONS TIME SERIES}

As mentioned in section II, spurious correlations may be detected if the time series be nonstationary, or is affected by trends. In such cases, direct calculation of the correlation exponent, the spectral density, the fractal dimensions, etc., do not yield reliable results. Our data sets are affected by some trends, such as the alternative current oscillation, the noise due to the electronic instruments, and the fluctuations of striation areas near the anode and cathode plates. Therefore, we must use detrended methods to distinguish the intrinsic fluctuations from the nonstationarity and trends.

Let us determine whether the data set has a sinusoidal trend or not. According to the MF-DFA method, the generalized Hurst exponents $h(q)$ in Eq. (4) are determined by analyzing the $\log -\log$ plots of $F_{q}(s)$ versus $s$ for each $q$. Using the rate of digitization in the experimental setup, 44100 sample/sec, one can simply change the unit of $s$ to seconds. It must be pointed out that, to infer the desired exponents and to avoid errors arising for small values of $s[19,20,30-32]$, we use the interval $s \geq 0.005$ sec in our analysis. The resolution of the recorded data in our setup is $1 / 44100 \sim 0.00023 \mathrm{sec}$. We use this interval throughout the paper, unless specified otherwise. Our investigation indicates that there is at least one crossover time scale in the log-log plots of $F_{q}(s)$ versus $s$ for every $q$. To determine its value, we use the following two criteria and combine their results:

(i) Based on the recent results by $[19,20]$ and $[42,43]$, every sinusoidal trend in the data causes some crossovers in the scaling function, $F_{q}(s)$, derived by the MF-DFA. The number of such crossovers depends on the size of data and the wavelength of the sinusoidal trends $[19,20]$. It is well known that the crossovers divide the fluctuations function into some regions that correspond to various scaling behaviors of $F_{q}(s)$ versus $s$, that are related to the competition between noise and trends $[19,20]$. To prove this statement and show how one can determine the value of crossovers, we generated numerically a time series which is a superposition of a correlated noise, namely, with the Hurst exponent $H=0.8$, and a sinusoidal trend with its period equal to $T=20 \mathrm{sec}$. As shown in Figure 2 , one sinusoidal trend is embedded in the data, and there are two crossovers at $s_{3} \sim 3 \mathrm{sec}$ and $s_{4} \sim 20 \mathrm{sec}$ in the fluctuation function. The larger crossover is directly associated with the period of the sinusoidal trend $[19,20]$.

The crossovers are also confirmed by resorting to the power spectrum of the data shown in the middle graph of the lower panel of Figure 2. One can observe that for $\nu>\nu_{2}=1 / s_{3} \sim 0.3 \mathrm{sec}^{-1}$, the scaling behavior of the power spectrum is the same as those for the correlated noise, indicating that in these scales the noise effect is dominant. Consequently, the scaling behavior of $F_{q}(s)$ for $s<s_{3}$ is very close to those of the correlated noise alone (see the upper panel of Figure 2).

Thereafter, we embedded ten sinusoidal trends with various frequencies in the original signal, and performed the same computations as shown in Figure 2. We found that, when one increases the number of sinusoidal trends in the original noise, then expects that the value of the crossover at large scales, namely, $s_{4}\left(\nu_{1}\right)$ ( which is related to the dominant embedded sinusoidal trends), will extend, as shown in Figure 2. In other words, we have an interval, i.e., $s \in\left[s_{2}, s_{4}\right]$, or in frequency space, $\nu \in\left[\nu_{1}, \nu_{3}\right]$, within which the scaling behavior of the fluctuation function changes smoothly. Therefore, we cannot determine an exact value for the crossover at large scales. Moreover, as shown in the upper panel of Figure 2, at time scales smaller than $s_{2}$ or for frequencies larger than $\nu_{3}$, the fluctuation function retrieves its noisy behavior. This observation depends on the amplitudes and frequencies of the embedded periodic trends. Therefore, the exact value of the crossover at small scale is not obvious in the spectral density and, hence, we use the following criteria.

ii) As discussed above the existence of many sinusoidal trends in our data set, we expect that a plot of $F_{q}(s)$ versus $s$ possess at least one crossover. This crossover divides $F_{q}(s)$ into two regions, as shown in the upper panel of Figure 3 (for instance, we take $q=2$ and use the data set with $I=50 \mathrm{~mA}$ ). To determine the value of the crossover, we introduce a $\Delta(s)$ function as:

$$
\Delta(s)=\sqrt{\left[F(s)-F_{\text {Linear }}(s)\right]^{2}}
$$

for each $q$, where $F(s)$ and $F_{\text {Linear }}(s)$ are the fluctuation functions for the original data and the filtered data produced by the F-DFA method (see below), respectively. In Figure 4, we plot $\Delta(s)$ as a function of $s$ for the plasma fluctuations. Hereafter, for convenience we omit the subscript $q$ and take $q=2$ unless expressed otherwise. The crossover occurs at $s_{\times} \sim 0.02 \mathrm{sec}$, corresponding to $\nu_{\times} \sim 50 \mathrm{sec}^{-1}$. Clearly, the fluctuation function for $s \leq s_{\times}$has the same scaling behavior as the noise without trends (see Figure 3).

As expressed in the last section, to cancel the sinusoidal trend in the MF-DFA, we have applied the F-DFA method. Indeed, we truncate the lowest frequencies up to the value that the regression of the linear fitting of the corresponding log-log plot of the fluctuation function for truncated data reaches 0.95 . It is well known that if we truncate the frequencies more than the necessary values for eliminating the crossover, some statistical properties of the underlying noise might be lost $[19,20,52]$. To eliminate the crossover scales, we need to remove approximately the first 400 terms of the Fourier expansion. Then, by the inverse Fourier transformation, the fluctuations without the sinusoidal trend are retrieved. The result is shown in Figure 5. The generalized Hurst exponent, the classical multifractal scaling exponents, and the 

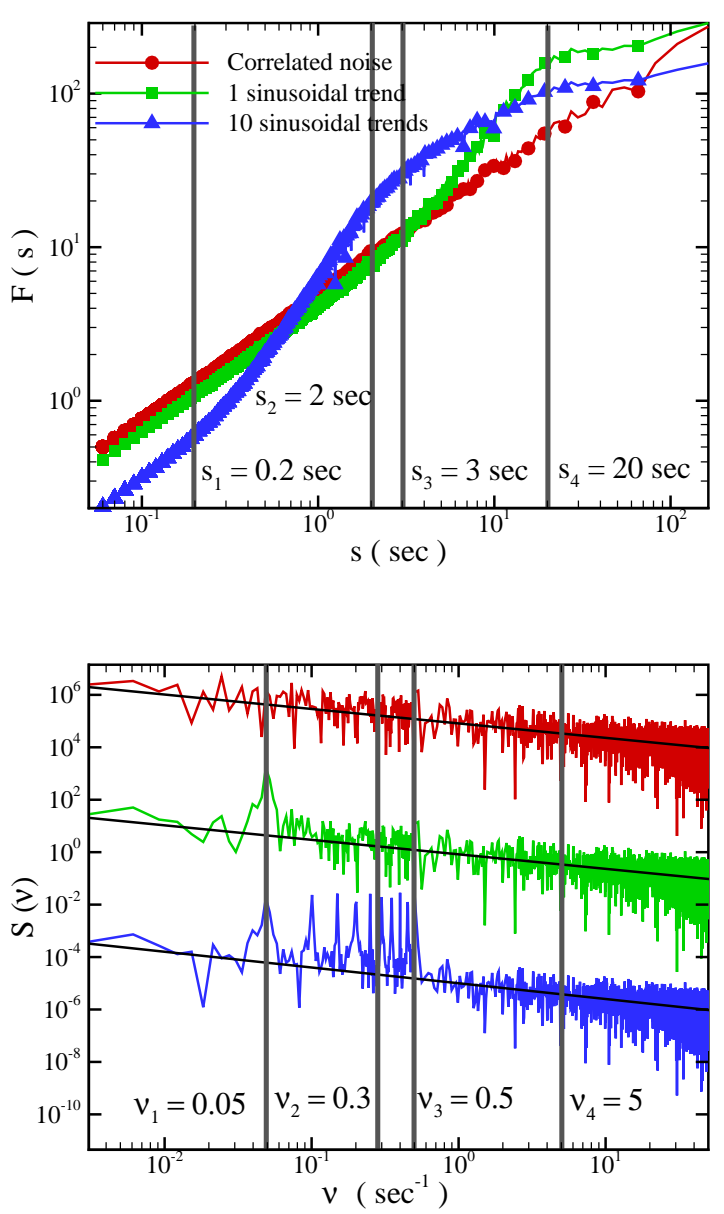

FIG. 2. Upper panel shows the fluctuations function for the correlated noise and its superposition with one and ten sinusoidal trends. Lower panel corresponds to their power spectrum. The curves, from top to bottom, correspond to the correlated noise, one and ten embedded sinusoidal trends in the noise, respectively. Oblique solid lines correspond to the scaling behavior of power spectrum of the clean correlated noise, namely, $\beta=0.6$ in $S(\nu) \sim \nu^{-\beta}$.

singularity spectrum for the data after the elimination of sinusoidal trends are illustrated in Figure 6. In addition, we estimated the errors at $1 \sigma$ confidence level for all the derived values reported in all the tables, using the common statistical method [64]. The cleaned time series is a multifractal process, as indicated by the strong $q$ dependence of the generalized Hurst exponents [53]. The $q$-dependence of the classical multifractal scaling exponent $\tau(q)$ has different behaviors for $q<0$ and $q>0$. For both positive and negative values of $q$, the value of slopes of $\tau(q)$ are indicated in Figure 6. As mentioned before for $q<0$, small fluctuations will be dominant in the fluctuation function, whereas for $q>0$ the large fluctuations represent the dominant effect in $F_{q}(s)$. From the statistical point of view, usually, for a multifractal anticorrelated series, namely, one with $H<0.5$, the value
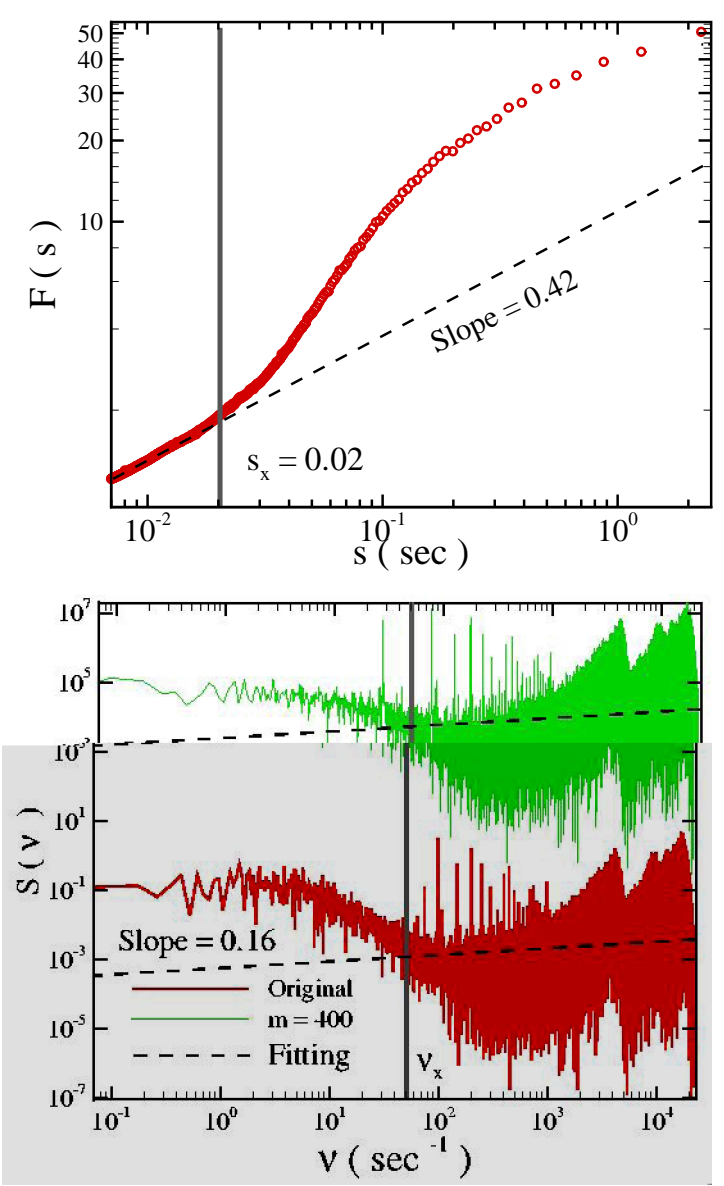

FIG. 3. Upper panel shows the crossover behavior of the $\log -\log$ plot of $F(s)$ versus $s$ for the original time series for $q=2.0$. Lower panel corresponds to the power spectrum of the original and cleaned data set. Oblique solid lines correspond to the scaling behavior of the power spectrum of the cleaned series, namely, $\beta=-0.16$ in $S(\nu) \sim \nu^{-\beta}$.

of $h(q)$ for $q<0$ is smaller than the generalized Hurst exponent for positive moments. This is due to the fact that, the number of the large fluctuations are statistically much more than the small fluctuations in the time series. In other words, the series is intermittent. The same circumstances arise for the plasma fluctuations. In the presence of free charge, every large deviations in the electrostatic equilibrium would be shielded by a cloud of oppositely charged particles $[49,65,66]$. Therefore, we expect the plasma fluctuations to be an anti-correlated series. The value of the Hurst exponent confirms that the data set is a stationary process. According to the MF-DFA results, all of the discharge current series behave as weak anti-correlated process. The value of the Hurst exponent and the classical multifractal exponent for $q=2$, in the region that the sinusoidal trend is not pronounced, calculated via the MF-DFA method are reported in Table I. The correlation and power spectrum exponents are given in Table II. 


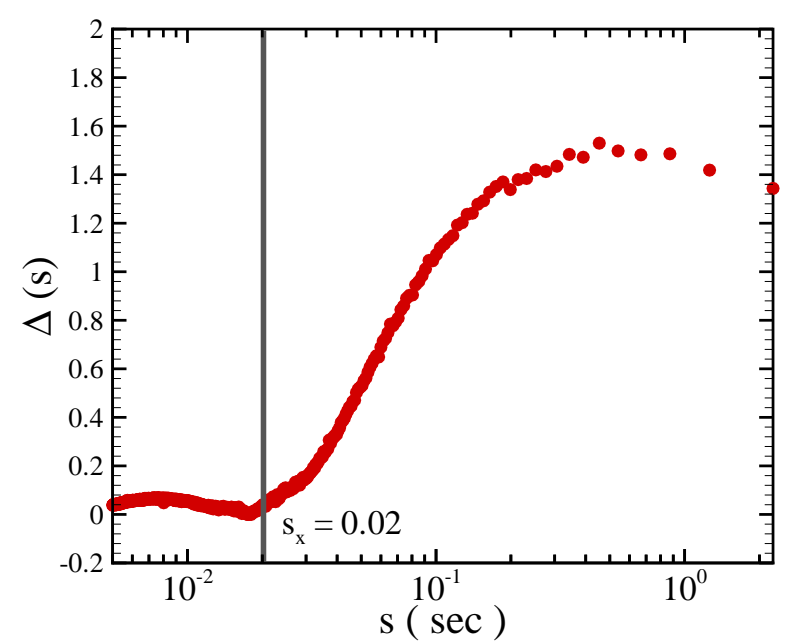

FIG. 4. The function $\Delta(s)$ for the plasma fluctuations versus $s$.

In spite of the power of the MF-DFA method, some cases encounter problems and the method yields inaccurate results. The DFA method can only determine a positive Hurst exponent, but yields an inaccurate result for the strongly anti-correlated recorded data, when $H \simeq 0$. To avoid this situation, one should use the integrated data. In that case the series is the so-called double profiled data set. The corresponding Hurst exponent is $H=\bar{H}-1$, where $\bar{H}$ is derived from the DFA method for the double profiled series [43,53].

Let us now discuss the finite size effect of the data set on the results. According to the recent analysis in Ref. [30-32], a deviation from the DFA results occurs in short records. The modified version of the MF-DFA should be used for such cases [30-32]. Usually, in the MF-DFA method, the deviation from a straight line in the log-log plot of Eq. (4) occurs for small scales $s$. The deviation limits the capability of DFA for determining the correct correlation behavior at very short scales, and in the regime of small $s$. The modified MF-DFA is defined as follows [30-32]

$$
F_{q}^{\bmod }(s)=F_{q}(s) \frac{\left\langle\left[F_{q}^{\text {shuf }}\left(s^{\prime}\right)\right]^{2}\right\rangle^{1 / 2} s^{1 / 2}}{\left\langle\left[F_{q}^{\text {shuf }}(s)\right]^{2}\right\rangle^{1 / 2} s^{1 / 2}} \quad\left(\text { for } s^{\prime} \gg 1\right)
$$

where $\left\langle\left[F_{q}^{\text {shuf }}(s)\right]^{2}\right\rangle^{1 / 2}$ denotes the usual MF-DFA fluctuation function [defined in Eq. (3)], averaged over several configurations of the shuffled data taken from the original time series, and $s^{\prime} \approx N / 40$. The values of the Hurst exponent obtained by the modified MF-DFA methods for the time series are reported in Table III. The maximum relative deviation of the Hurst exponent, which is computed by the modified MF-DFA, relative to the MF-DFA for the original data, is approximately $2.7 \%$. Moreover, Figure 7 shows a comparison between the generalized Hurst

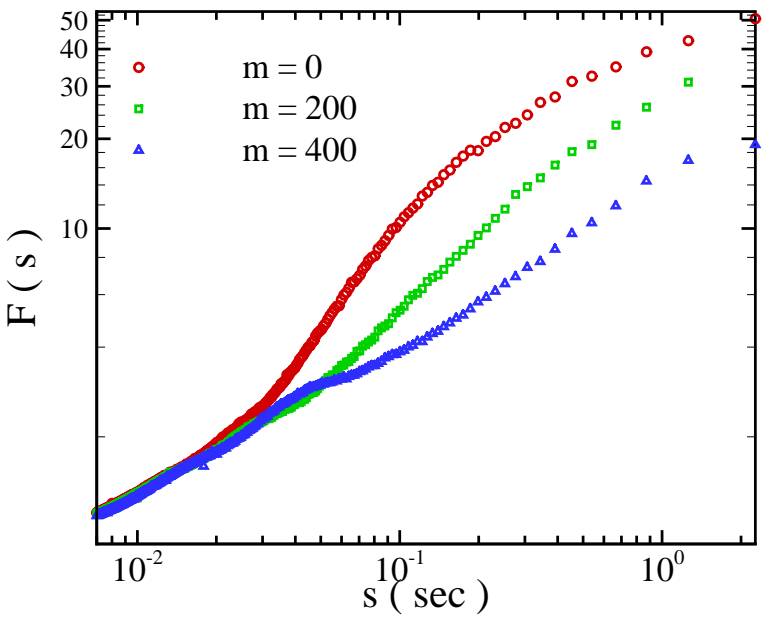

FIG. 5. The MF-DFA functions $F_{q}(s)$ in terms of the time scale $s$, in a log-log plot. The original time series, $m=0$, the truncation of the first 200, $m=200$, and 400 terms, $m=400$. This plot is for a typical value of the electrical discharge current intensity, $I=50 \mathrm{~mA}$.

exponent, derived by the common MD-DFA1 and modified MF-DFA. It indicates that the modified MF-DFA is consistent with the MF-DFA at $1 \sigma$ confidence level for various moments.

By inspecting the log-log plot of the fluctuation function versus $s$ (i.e., $F_{2}(s)=\mathcal{C}_{\mathcal{H}} s^{H}$ ), we find the dependence of its amplitude on the electrical discharge current. Indeed, Ref. [42] showed that this amplitude is given by

$$
\begin{aligned}
\mathcal{C}_{\mathcal{H}}^{2}= & \frac{\sigma^{2}}{(2 H+1)}-\frac{4 \sigma^{2}}{2 H+2} \\
& +3 \sigma^{2}\left(\frac{2}{H+1}-\frac{1}{2 H+1}\right) \\
& \quad-\frac{3 \sigma^{2}}{(H+1)}\left(1-\frac{1}{(H+1)(2 H+1)}\right)
\end{aligned}
$$

where $\sigma^{2}=\left\langle x(i)^{2}\right\rangle$ is the variance of the data. Figure 8 shows the value of the amplitude as a function of the current.

\section{SEARCH FOR ORIGIN OF MULTIFRACTALITY IN THE DATA SET}

Due to the strong $q$ dependence of the generalized Hurst exponent and the distinct slopes $\tau(q)$ for the various moments, the remaining data set, after the elimination of the sinusoidal trends, has multifractal properties. In this section we search for the source of the multifractality. In general, two different types of multifractality in time series may exist:

(1) Multifractality due to the fatness of the probability density function (PDF) of the time series, in compari- 

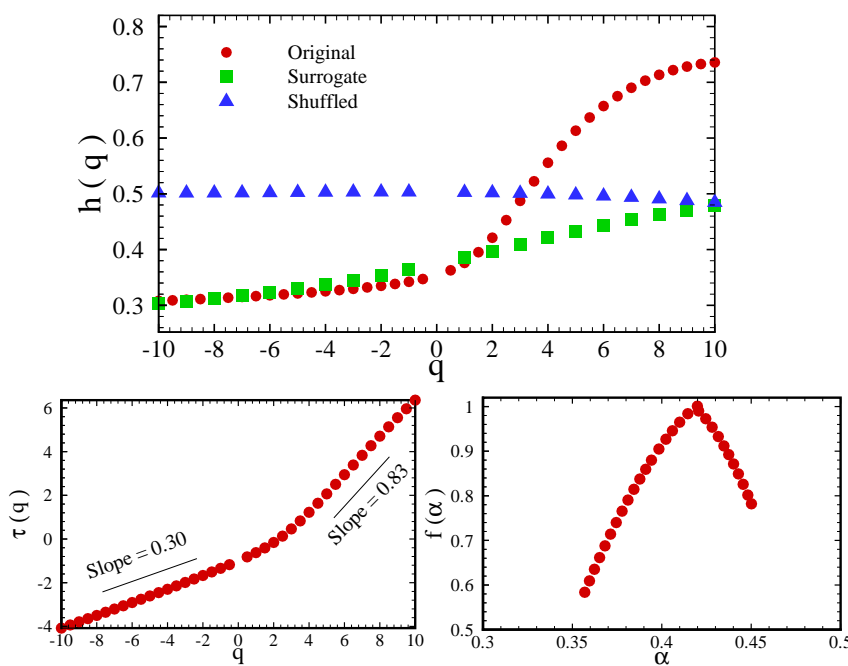

FIG. 6. Upper panel shows the generalized Hurst exponent versus $q$ for the original, the surrogate, and the shuffled series without the sinusoidal trend. Lower panel left and right correspond to the classical multifractal scaling exponent and the singularity spectrum $f(\alpha)$ for the data set at $I=50 \mathrm{~mA}$, respectively.

son to a Gaussian PDF. In this case, the multifractality cannot be removed by shuffling the series, because the correlations in the data set are affected by the shuffling, while the PDF of the series is invariant.

(2) Multifractality due to different types of correlations in the small and large scale fluctuations. In this case, the data may have a PDF with finite moments, e.g., a Gaussian distribution. Thus, the corresponding shuffled time series will exhibit monofractal scaling, since all the correlations are destroyed by the shuffling procedure. If both kinds of multifractality are present, the shuffled series will exhibit weaker multifractality than the original series.

The easiest way to distinguish the type of multifractality is by analyzing the corresponding shuffled and surrogate time series. The shuffling of the time series destroys the correlation. Therefore, if the multifractality only belongs to the correlation, we should find $h_{\text {shuf }}(q)=0.5$. The multifractality due to the fatness of the PDF series is not affected by the shuffling procedure. On the other hand, to determine whether the multifractality is due to the broadness or fatness of the PDF, the surrogate data are used [67]. If multifractality in the time series is due to a broad PDF, $h(q)$ for surrogate data will be independent of $q$. If both kinds of multifractality are present in fluctuations, the shuffled and surrogate series will exhibit weaker multifractality than the original one. The utility of the two tests was first demonstrated by Ivanov et al. [68-70].

To check the nature of the multifractality, the we compare the fluctuation function $F_{q}(s)$ for the original series (after removal of the sinusoidal trends) with the results

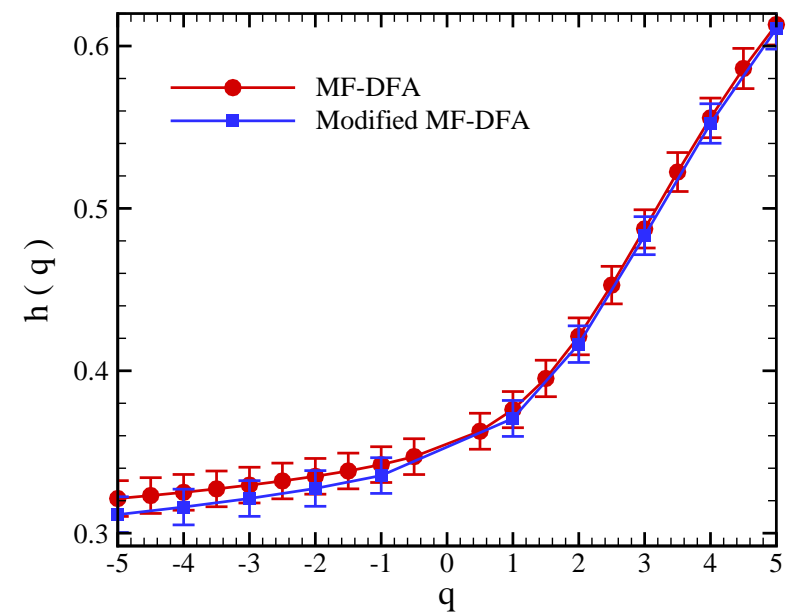

FIG. 7. Comparison between the modified and commonly used MF-DFA for a typical value of the electrical-discharge current intensity, $I=50 \mathrm{~mA}$.

for the corresponding shuffled, $F_{q}^{\text {shuf }}(s)$ and that of the surrogate series, $F_{q}^{\text {sur }}(s)$. The differences between the two fluctuation functions with the original one, directly indicate the presence of the correlations or broadness of the PDF in the original series. The differences can be observed in plots of $F_{q}(s) / F_{q}^{\text {shuf }}(s)$ and $F_{q}(s) / F_{q}^{\text {sur }}(s)$ versus $s$ [53]. Since the anomalous scaling due to a broad PDF affects $F_{q}(s)$ and $F_{q}^{\text {shuf }}(s)$ in the same way, only multifractality due to the correlations will be observed in $F_{q}(s) / F_{q}^{\text {shuf }}(s)$. The scaling behavior of the two ratios are given by

$$
\begin{aligned}
& F_{q}(s) / F_{q}^{\text {shuf }}(s) \sim s^{h(q)-h_{\text {shuf }}(q)}=s^{h_{\text {cor }}(q)} \\
& F_{q}(s) / F_{q}^{\text {sur }}(s) \sim s^{h(q)-h_{\text {sur }}(q)}=s^{h_{\mathrm{PDF}}(q)}
\end{aligned}
$$

If only the fatness of the PDF is responsible for the multifractality, one should obtain, $h(q)=h_{\text {shuf }}(q)$, and, $h_{\text {cor }}(q)=0$. On the other hand, deviations from $h_{\text {cor }}(q)=0$ indicates the presence of correlations, while the $q$ dependence of $h_{\text {cor }}(q)$ indicates that multifractality is due to the correlation. If both the distribution and correlation multifractality are present, both $h_{\text {shuf }}(q)$ and $h_{\text {sur }}(q)$ will depend on $q$.

The $q$ dependence of the exponent $h(q)$ for the original, surrogate, and shuffled time series are shown in Figures 6. The $q$ dependence of $h_{\mathrm{PDF}}$ shows that the multifractality nature of the time series is almost due to the correlation. However, the value of $h_{\mathrm{PDF}}(q)$ is deviated from zero which confirms the multifractality due to the broadness of the PDF is much weaker than the multifractality due to the correlation. The deviation of $h_{\text {sur }}(q)$ and $h_{\text {shuf }}(q)$ from $h(q)$ may be determined by using the $\chi^{2}$ test:

$$
\chi_{\diamond}^{2}=\sum_{i=1}^{N} \frac{\left[h\left(q_{i}\right)-h_{\diamond}\left(q_{i}\right)\right]^{2}}{\sigma\left(q_{i}\right)^{2}+\sigma_{\diamond}\left(q_{i}\right)^{2}}
$$




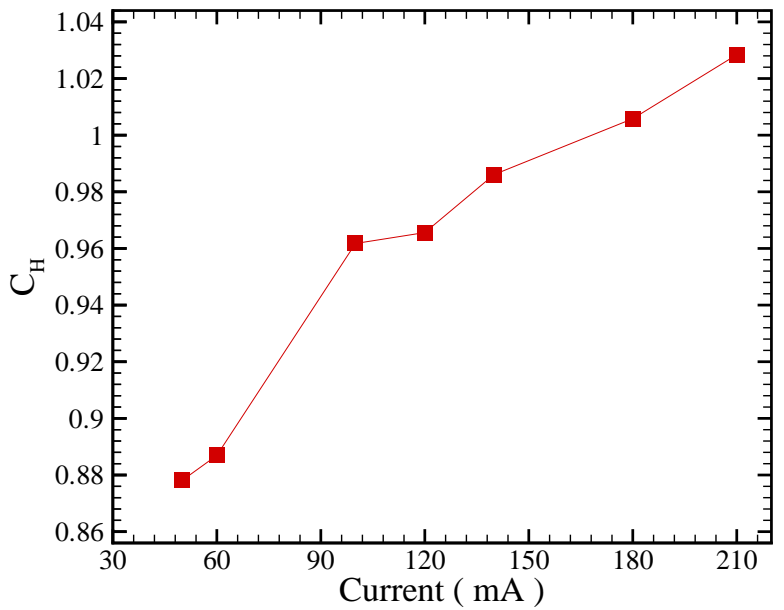

FIG. 8. The value of $\mathcal{C}_{\mathcal{H}}$ as a function of the discharge current intensity.

where the symbol $\diamond$ can be replaced by "sur" and "shuf", in order to determine the confidence level of $h_{\text {sur }}$ and $h_{\text {shuf }}$, the generalized Hurst exponents of the original series, respectively. The value of the reduced chi-square, $\chi_{\nu}^{2}=\chi^{2} / \mathcal{N}$ where $\mathcal{N}$ is the number of degree of freedom, for the shuffled and surrogate time series are shown in the upper panel of Figure 9. On the other hand, the lower panel of Figure 9 illustrates the width of the singularity spectrum, $f(\alpha)$, i.e., $\Delta \alpha=\alpha\left(q_{\min }\right)-\alpha\left(q_{\max }\right)$, for the original, surrogate, and shuffled data set. These values also confirm that the multifractality due to the correlations is dominant [55].

The values of the generalized Hurst exponent $h(q=$ $2.0)$, and the multifractal scaling $\tau(q=2)$ for the original, shuffled, and surrogate of the discharge fluctuation obtained with the MF-DFA method are reported in Table I.

\section{CONCLUSION}

Discharge current fluctuations in plasma are affected by many factors. From a statistical point of view, and in order to understand the complexity of the fluctuations, we applied a robust method, such as the detrending fractal analysis to infer the complexity and multifractal features of the underlying plasma fluctuations. In the presence of nonstationarity, non-detrending methods will be encountered with some challenges, such that they yield wrong or at least inaccurate results. Multifractal detrended fluctuation analysis is well established for investigating noisy time series, and can be used to gain deeper insight into the processes that occur in nonstationary dynamical systems, such as electrical discharge current.
TABLE I. Values of $H=h(q=2)$ and the classical multifractal scaling exponents for $q=2.0$ for the original, surrogate and shuffled data set and different electrical currents, obtained by the MF-DFA.

\begin{tabular}{|c|c|c|c|}
\hline \multirow[t]{2}{*}{ Sample } & & $H$ & $\tau$ \\
\hline & Original & $0.42 \pm 0.01$ & $-0.16 \pm 0.02$ \\
\hline \multirow[t]{3}{*}{$50 \mathrm{~mA}$} & Surrogate & $0.40 \pm 0.01$ & $-0.21 \pm 0.02$ \\
\hline & Shuffled & $0.50 \pm 0.01$ & $0.004 \pm 0.020$ \\
\hline & Original & $0.45 \pm 0.01$ & $-0.10 \pm 0.02$ \\
\hline \multirow[t]{3}{*}{$60 \mathrm{~mA}$} & Surrogate & $0.42 \pm 0.01$ & $-0.16 \pm 0.02$ \\
\hline & Shuffled & $0.50 \pm 0.01$ & $0.002 \pm 0.020$ \\
\hline & Original & $0.37 \pm 0.01$ & $=0.25 \pm 0.02$ \\
\hline \multirow[t]{3}{*}{$100 \mathrm{~mA}$} & Surrogate & $0.36 \pm 0.01$ & $-0.28 \pm 0.02$ \\
\hline & Shuffled & $0.49 \pm 0.01$ & $0.00 \pm 0.02$ \\
\hline & Original & $0.38 \pm 0.01$ & $-0.23 \pm 0.02$ \\
\hline \multirow[t]{3}{*}{$120 \mathrm{~mA}$} & Surrogate & $0.36 \pm 0.01$ & $-0.28 \pm 0.02$ \\
\hline & Shuffled & $0.50 \pm 0.01$ & $0.00 \pm 0.02$ \\
\hline & Original & $0.41 \pm 0.01$ & $-0.17 \pm 0.02$ \\
\hline \multirow[t]{3}{*}{$140 \mathrm{~mA}$} & Surrogate & $0.40 \pm 0.01$ & $-0.21 \pm 0.02$ \\
\hline & Shuffled & $0.50 \pm 0.01$ & $0.00 \pm 0.02$ \\
\hline & Original & $0.45 \pm 0.01$ & $-0.09 \pm 0.02$ \\
\hline \multirow[t]{3}{*}{$180 \mathrm{~mA}$} & Surrogate & $0.40 \pm 0.01$ & $-0.20 \pm 0.02$ \\
\hline & Shuffled & $0.50 \pm 0.01$ & $-0.003 \pm 0.020$ \\
\hline & Original & $0.48 \pm 0.01$ & $=-0.04 \pm 0.02$ \\
\hline \multirow[t]{2}{*}{$210 \mathrm{~mA}$} & Surrogate & $0.44 \pm 0.01$ & $-0.13 \pm 0.02$ \\
\hline & Shuffled & $0.51 \pm 0.01$ & $0.01 \pm 0.02$ \\
\hline
\end{tabular}

TABLE II. Values of the correlation and power spectrum exponents for the original data set with different electrical currents, obtained by the MF-DFA.

\begin{tabular}{|c|c|c|}
\hline \hline Sample & $\gamma$ & $\beta$ \\
\hline $50 \mathrm{~mA}$ & $1.16 \pm 0.02$ & $-0.16 \pm 0.02$ \\
\hline $60 \mathrm{~mA}$ & $1.10 \pm 0.02$ & $-0.10 \pm 0.02$ \\
\hline $100 \mathrm{~mA}$ & $1.26 \pm 0.02$ & $-0.26 \pm 0.02$ \\
\hline $120 \mathrm{~mA}$ & $1.24 \pm 0.02$ & $-0.24 \pm 0.02$ \\
\hline $140 \mathrm{~mA}$ & $1.18 \pm 0.02$ & $-0.18 \pm 0.02$ \\
\hline $180 \mathrm{~mA}$ & $0.10 \pm 0.02$ & $-0.10 \pm 0.02$ \\
\hline $210 \mathrm{~mA}$ & $1.04 \pm 0.02$ & $-0.04 \pm 0.02$ \\
\hline \hline
\end{tabular}

TABLE III. Values of Hurst exponent using the regular MF-DFA and modified MF-DFA for data sets.

\begin{tabular}{|c|c|c|}
\hline \hline Sample & $H$ & $H_{\mathrm{Mod}}$ \\
\hline $50 \mathrm{~mA}$ & $0.42 \pm 0.01$ & $0.42 \pm 0.01$ \\
\hline $60 \mathrm{~mA}$ & $0.45 \pm 0.01$ & $0.45 \pm 0.01$ \\
\hline $100 \mathrm{~mA}$ & $0.37 \pm 0.01$ & $0.38 \pm 0.01$ \\
\hline $120 \mathrm{~mA}$ & $0.38 \pm 0.01$ & $0.37 \pm 0.01$ \\
\hline $140 \mathrm{~mA}$ & $0.41 \pm 0.01$ & $0.42 \pm 0.01$ \\
\hline $180 \mathrm{~mA}$ & $0.45 \pm 0.01$ & $0.46 \pm 0.01$ \\
\hline $210 \mathrm{~mA}$ & $0.48 \pm 0.01$ & $0.48 \pm 0.01$ \\
\hline \hline
\end{tabular}



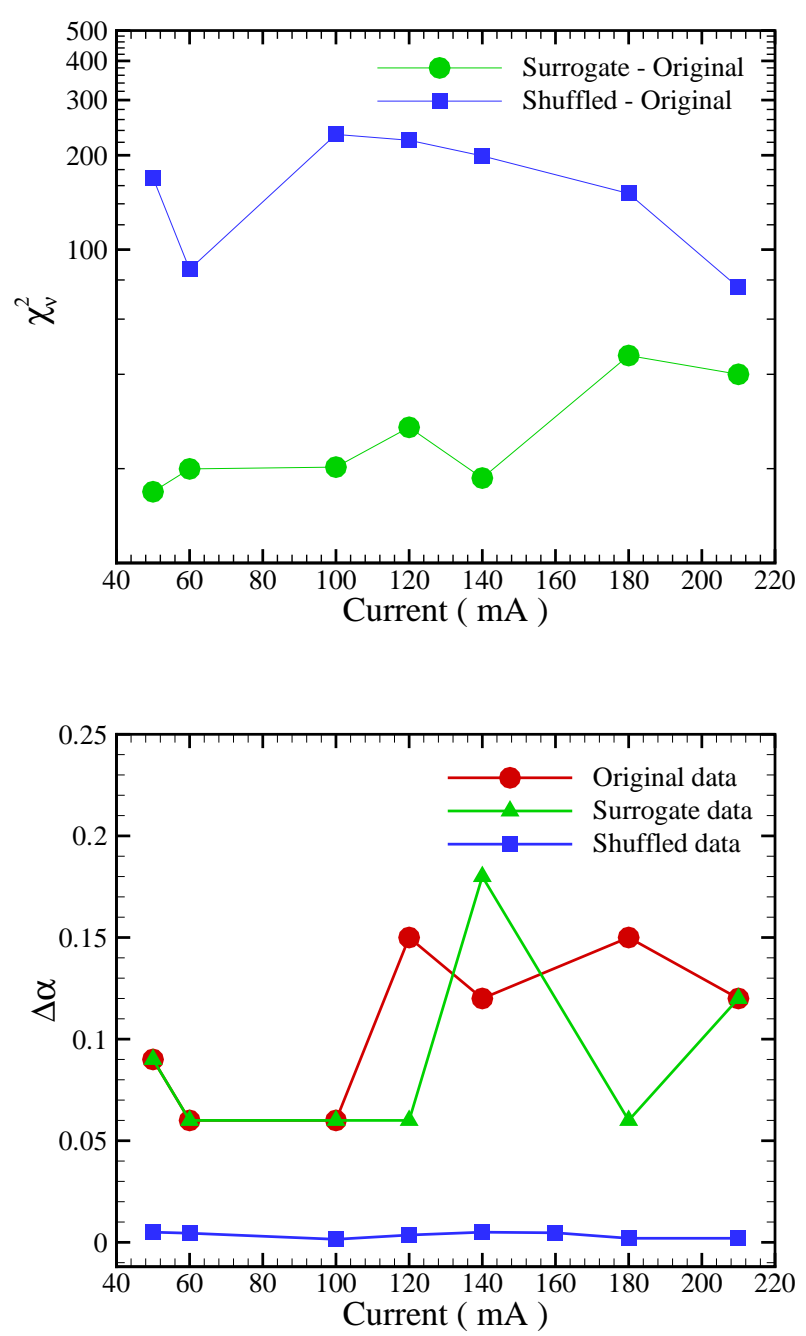

FIG. 9. Upper panel shows to the values of $\chi_{\nu}^{2}$ for the data sets. Lower panel shows the width of the singularity strength, $\Delta \alpha$, for the original, surrogate and shuffled data sets in various electrical discharge currents, obtained by the MF-DFA.

We showed that the MF-DFA results for the time series for various electrical currents have one crossover time scale, $s_{\times}$. This crossover time scale is about $s_{\times} \sim 0.02$ second, and is related to the sinusoidal trends. The crossover time scale which discriminates the noise and trends intervals can be potentially related to the coherent time scale in turbulent plasma. Plasma fluctuations are not affected by external factors within time scale. To minimize the effect of trends and produce clean data set for further investigation, we applied the Fourier detrended fluctuation analysis to the data sets. Indeed, after applying the F-DFA, the data set without sinusoidal trends is recovered, and the spurious behavior in the MDDFA results disappear. Applying the MF-DFA method on the cleaned data set demonstrated that the discharge current fluctuations are stationary time series.
According to the value of the Hurst exponent, computed by the MF-DFA method, all the discharge current time series behave as weak anti-persistent processes. These findings can be interpreted as follows: in the presence of free charges, every large deviation from the electrostatic equilibrium is shielded by a cloud of oppositelycharged particles $[49,65,66]$. This also may be related to the fast dissipation of turbulent kinetic energy in plasma. Our results also confirmed that the multifractality nature is a global property of various plasma data based on different experimental setups [11]. We found that Hurst exponent and multifractality nature based on singularity spectrum didn't depend on the discharge current intensity (see Table III and Figure 9). This result indicates that increasing the amount of charged particles at least in our experimental setup almost don't alter the statistical properties of the plasma fluid. But it is interesting to extend these analysis to a broader set of plasma data in various working pressure and check their statistical properties. The $q$-dependence of $h(q)$ and $\tau(q)$ indicated that the data sets have multifractal properties.

The value of $h(q)$ for $q>0$ is larger than the same quantity for $q<0$, indicates that the number of large fluctuations are statistically larger than the small fluctuations in the time series. Our results show that, amplitude of the fluctuation function $\mathcal{C}_{\mathcal{H}}$ is a monotonous function in terms of discharge current intensity. This demonstrate that by increasing the current intensity, plasma instability will be occurred [13] and consequently one has large variance for data set (see Figure 8).

In order to recognize the nature of the multifractality, we compared the generalized Hurst exponent of the original time series with those of the shuffled and surrogate ones. The comparison indicated that the multifractality due to the correlations makes more significant contribution than the broadness of the probability density function of the current fluctuations.

Acknowledgements The authors are grateful to the anonymous referee for his/her useful comments. The experimental part of this research was supported by Tabriz University. We also thank M. Sahimi for a critical reading of the manuscript.

[1] A. Garscadden and K. G. Emeleus, Proc. Phys. Soc. 79,6 (1962).

[2] W. X. Ding, T. Klinger and A. Piel, Phys. Lett. A 222,409 (1996).

[3] J. Qin, L. Wang, D. P. Yuan, P. Gao, and B. Z. Zhang, Phys. Rev. Lett. 63, 163 (1989).

[4] Z. Donko and L. Szalia, Chaos, Solitons, Fractals 7, 5 (1996).

[5] T. Gyergyek, Plasma Phys. and Cont. Fusion 41,175 
(1999).

[6] C. Franck, T. Klinger and A. Piel, Phys. Lett. A, 259,152 (1999).

[7] C. Letellier, O. Menard, T. Klinger, A. Piel and G. Bonohomme, Physica D 156,169 (2001).

[8] V.P. Budaev, E. Dufkova, S. Nanobashvili, V. Weinzettl and J. Zajac, 32nd EPS Conference on Plasma Phys. Tarragona, 27 June - 1 July 2005 ECA Vol.29C, P-5.019 (2005).

[9] J. Zajac, E. Dufkova, V. Weinzettl, V. P. Budaev and S. Nanobashvili, Czechoslovak Journal of Physics, Volume 55, No. 12 (2005)

[10] B. van Milligen, C. Hidalgo, R. Balbin, E. Sanchez, I. Garcia-Cortes, and M. A. Pedrosa, Phys. Rev. Lett.83, 3653 - 3656 (1999).

[11] V.P. Budaev, Physica A: Statistical Mechanics and its Applications Volume 344, Issues 1-2 (2004).

[12] V.P. Budaev, S.Takamura and N. Ohno, 32nd EPS Conference on Plasma Phys. Tarragona, 27 June - 1 July 2005 ECA Vol.29C, P-4.050 (2005).

[13] V. Weinzettl et al, 32nd EPS Conf. on Pl. Phys. Taragona, P4.006 (2005).

[14] B. A. Carreras et. al., Phys. Plasmas 7, 3278 (2000).

[15] A. Dinklage T. Klinger G.Marx L. Schweikhard (Editors)" Plasma Physics Confinement, Transport and Collective Effects" (Springer, Berlin Heidelberg 2005).

[16] F. Sigeneger and K. Winkler, Applied Physics 19, 211 (2002).

[17] A. V. Isupov and I. M. Ulanov, High Temprature 43, 169 (2005).

[18] I. Denysenko et al. Physics of Plasma 13, 073507 (2006).

[19] K. Hu, P. Ch. Ivanov, Z. Chen, P. Carpena and H. E. Stanley, Phys. Rev. E 64, 011114 (2001).

[20] Zhi Chen, Plamen Ch. Ivanov, Kun Hu, H. Eugene Stanley, Phys. Rev. E 65, 041107 (2002).

[21] J. Feder, Fractals (Plenum Press, New York, 1988).

[22] A. L. Barabási and T. Vicsek, Phys. Rev. A 44, 2730 (1991).

[23] H. O. Peitgen, H. Jürgens and D. Saupe, "Chaos and Fractals" (Springer-Verlag, New York,1992), Appendix B.

[24] E. Bacry, J. Delour and J. F. Muzy, Phys. Rev. E 64, 026103 (2001).

[25] J. F. Muzy, E. Bacry and A. Arneodo, Int. J. Bifurcation and Cheos 4(2), 245 (1994).

[26] J. F. Muzy, E. Bacry and A. Arneodo, Phys. Rev. Lett. 67, 3515 (1991).

[27] C. K. Peng, S. V. Buldyrev, S. Havlin, M. Simons, H. E. Stanley and A. L. Goldberger, Phys. Rev. E 49, 1685 (1994).

[28] S. M. Ossadnik, S. B. Buldyrev, A. L. Goldberger, S. Havlin, R. N. Mantegna, C. K. Peng, M. Simons and H. E. Stanley, Biophys. J. 67, 64 (1994).

[29] M. S. Taqqu, V. Teverovsky and W. Willinger, Fractals 3, 785 (1995).

[30] J. W. Kantelhardt, E. Koscielny-Bunde, H. H. A. Rego, S. Havlin and A. Bunde, Physica A 295, 441 (2001).

[31] Radhakrishnan Nagarajan and Rajesh G. Kavasseri, Chaos, Solitons and Fractals 26, 777784 (2005).

[32] Radhakrishnan Nagarajan and Rajesh G. Kavasseri, Physica A 354, 182198 (2005).
[33] C. K. Peng, S. Havlin, H. E. Stanley and A. L. Goldberger, Chaos 5, 82 (1995).

[34] P. Ch. Ivanov, A. Bunde, L. A. N. Amaral, S. Havlin, J. Fritsch-Yelle, R. M. Baevsky, H. E. Stanley and A. L. Goldberger, Europhys. Lett. 48, 594 (1999).

[35] A. Bunde, S. Havlin, J. W. Kantelhardt, T. Penzel, J. H. Peter and K. Voigt, Phys. Rev. Lett. 85, 3736 (2000).

[36] R. N. Mantegna and H. E. Stanley, "An Introduction to Econophysics" (Cambridge University Press, Cambridge, 2000)

[37] Y. Liu, P. Gopikrishnan, P. Cizeau, M. Meyer, C. K. Peng and H. E. Stanley, Phys. Rev. E 60, 1390 (1999).

[38] N. Vandewalle, M. Ausloos and P. Boveroux, Physica A 269, 170 (1999).

[39] G. R. Jafari, M. S. Movahed, S. M. Fazeli, M. Reza Rahimi Tabar, S. F. Masoudi, J. Stat. Mech, P06008 (2006).

[40] P. C. Ivanov, A. Yuen, B. Podobnik, Y. Lee, Phys. Rev. E 69, 056107 (2004).

[41] G. R. Jafari, M. Sadegh Movahed, P. Noroozzadeh, A. Bahraminasab, Muhammad Sahimi, F. Ghasemi, M. Reza Rahimi Tabar, International Journal of Modern Physics C Vol. 18, No. 11, 16891697(2007).

[42] M. Sadegh Movahed and Evalds Hermanis, Physica A 387, 915 (2008).

[43] M. Sadegh Movahed, G. R. Jafari, F. Ghasemi, S. Rahvar and M. Rahimi Tabar, J. Stat. Mech, P02003 (2006).

[44] M. Sadegh Movahed, F. Ghasemi, S. Rahvar and M. Rahimi Tabar, arXiv:astro-ph/0602461

[45] G. R. Jafari, P. Pedram, L. Hedayatifar, J. Stat. Mech, P04012 (2007).

[46] G. R. Jafari, P. Pedram, K. Ghafoori Tabrizi, AIP Conf. Proc. 889, 310 (2007).

[47] Heather D. Jennings, Plamen Ch. Ivanov, Allande M. Martins, P. C. da Silva and G.M. Viswanathan, Physica A 336, 585 (2004).

[48] H. E. Hurst, R. P. Black and Y. M. Simaika, " Longterm storage. An experimental study" (Constable, London, 1965).

[49] Michael A. Lieberman and Allan J. Lichtenberg, "Principles of Plasma Discharges and Materials Processing",(Wiley-Interscience, 1994).

[50] S. V. Buldyrev, A. L. Goldberger, S. Havlin, R. N. Mantegna, M. E. Matsa, C. K. Peng, M. Simons and H. E. Stanley, Phys. Rev. E 51, 5084 (1995).

[51] R. Nagarajan and R. G. Kavasseri, Int. Journal of Bifurcations and Chaos, 15, no.2, (2005).

[52] C. V. Chianca, A. Ticona and T. J. P. Penna, Physica A 357, 447 (2005).

[53] J. W. kantelhardt, S. A. Zschiegner, E. Koscielny-Bunde, A. Bunde, S. Havlin and H. E. Stanley, Physica A 316, 87 (2002).

[54] A. Eke, P. Herman, L. Kocsis and l. R. Kozak, Physiol. Meas. 23, R1 (2002).

[55] P. Oświęcimka, Jaroslaw Kwapien and S. Drozdz, Phys. Rev. E 74, 016103 (2006).

[56] J. W. Cooley and J.W. Tukey, Mathematics of Computation, 19, 297 (1965).

[57] E. Koscielny-Bunde, H. E. Roman, A. Bunde, S. Havlin and H. J. Schellnhuber, Phil. Mag. B 77, 1331 (1998).

[58] E. Koscielny-Bunde, A. Bunde, S. Havlin, H. E. Roman, 
Y. Goldreich, and H. J. Schellnhuber, Phys. Rev. Lett. 81729 (1998).

[59] Dean Prichard and J. Theiler, Phys. Rev. Lett 73, 951 (1994).

[60] P.F. Panter, "Modulation, Noise and Specteral Analysis Applied to Information Transmission", ( New York,Mc Graw Hil, 1965).

[61] Tomoya Suzukia, Tohru Ikeguchib and Masuo Suzuki, Physica D: Nonlinear Phenomena Volume 231, pp. 108115 (2007).

[62] T. Schreibor and A. Schmitz, Physica D: Nonlinear Phenomena Volume 142, pp. 346382 (2000).

[63] A. Galka, "Topics in Nonlinear Time Series Analysis with Implications for EEG Analysis" (World Scientific, Cambrige university press, 2000).

[64] G. L. Squires, "Practical Physics", (Cambridge University Press, Cambridge, 1985), Third edition.

[65] Francis F. Chen, "Introduction to Plasma Physics", (Plenum Press New York 1974).

[66] Ferdinand F. Cap, "Handbook on Plasma Instabilities",( Academic Press, New York 1976).

[67] J. Theiler, S Eubank, A. Longtin and J. D. Farmer, Physica D 58, 77 (1992).

[68] P. C. Ivanov et al. Nature,399,44 (1999).

[69] P.C. Ivanov et al. Chaos 11 (3), 641 (2001).

[70] P. C. Ivanov et al. Nature 383, (6598) 323 (1996). 